\title{
Alterstice
}

Revue internationale de la recherche interculturelle

International Journal of Intercultural Research

Revista International de la Investigacion Intercultural

\section{Manifestations du fait religieux dans les institutions de santé de première ligne au Québec}

\section{Josiane Le Gall, Spyridoula Xenocostas et Sophie Lemoyne-Dessaint}

Volume 2, numéro 2, 2012

Regards pluriels sur les interventions sociales et de santé en contexte de diversité

URI : https://id.erudit.org/iderudit/1077562ar

DOI : https://doi.org/10.7202/1077562ar

Aller au sommaire du numéro

Éditeur(s)

Alterstice

ISSN

1923-919X (numérique)

Découvrir la revue

Citer cet article

Le Gall, J., Xenocostas, S. \& Lemoyne-Dessaint, S. (2012). Manifestations du fait religieux dans les institutions de santé de première ligne au Québec. Alterstice, 2(2), 23-34. https://doi.org/10.7202/1077562ar
Résumé de l'article

En dépit de la multiplication de nouveaux groupes religieux au Québec au cours des dernières décennies, l'impact du pluralisme religieux sur l'organisation et la prestation des services de santé demeure peu documenté dans le contexte québécois. Dans cet article, à partir des données recueillies lors d'une recherche sur les défis et enjeux posés par la diversité religieuse dans l'intervention de première ligne, nous examinons les manifestations de la religion dans la relation de soins : quels sont les types de demandes des patients en matière religieuse ? Quelles sont les difficultés attribuables à la diversité religieuse ? Quelles sont les réponses des intervenants à ces demandes ou cas difficiles? 


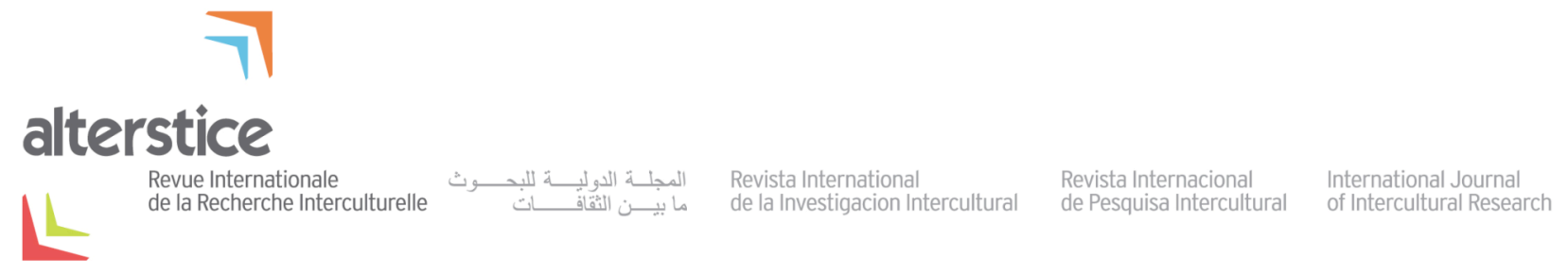

ARTICLE THÉMATIQUE

\title{
Manifestations du fait religieux dans les institutions de santé de première ligne au Québec
}

Josiane Le Gall ${ }^{1,2}$, Spyridoula Xenocostas ${ }^{2}$ et Sophie Lemoyne-Dessaint ${ }^{2}$

\section{Résumé}

En dépit de la multiplication de nouveaux groupes religieux au Québec au cours des dernières décennies, l'impact du pluralisme religieux sur l'organisation et la prestation des services de santé demeure peu documenté dans le contexte québécois. Dans cet article, à partir des données recueillies lors d'une recherche sur les défis et enjeux posés par la diversité religieuse dans l'intervention de première ligne, nous examinons les manifestations de la religion dans la relation de soins : quels sont les types de demandes des patients en matière religieuse? Quelles sont les difficultés attribuables à la diversité religieuse? Quelles sont les réponses des intervenants à ces demandes ou cas difficiles?

\author{
Rattachement des auteures \\ ${ }^{1}$ Département d'anthropologie, Université de Montréal, Montréal, Canada; ${ }^{2}$ CSSS de la Montagne, Montréal, \\ Canada
}

\section{Correspondance}

j.legall@ssss.gouv.qc.ca

\section{Mots clés}

religion; première ligne; CSSS; intervention

\section{Pour citer cet article :}

Le Gall, J., Xenocostas, S. et Lemoyne-Dessaint, S. (2012). Manifestations du fait religieux dans les institutions de santé de première ligne au Québec. Alterstice, 2(2), 23-34. 


\section{Introduction}

En 2006, parmi les controverses qui éclatèrent dans les médias québécois au sujet d'accommodements raisonnables, plusieurs touchaient le milieu des soins, comme l'illustrent les exemples suivants: la décision d'une clinique d'urgence sans rendez-vous d'un $\mathrm{CLSC}^{1}$ lavallois de traiter un patient juif orthodoxe avant plusieurs autres patients afin qu'il puisse retourner chez lui avant le coucher du soleil et ainsi respecter le sabbat; l'interdiction faite aux hommes d'assister à des cours prénatals d'un CLSC montréalais afin de répondre à la demande de certaines usagères, qui, pour des motifs religieux, refusent d'assister à ces cours en présence d'hommes; le décès d'un Témoin de Jéhovah dans un hôpital après avoir refusé une transfusion sanguine ${ }^{2}$. Comme l'a souligné le rapport rédigé à la suite de la Commission de consultation sur les pratiques d'accommodement reliées aux différences culturelles (CCPARDC), ces cas, largement médiatisés, reposent fréquemment comme dans les exemples précédents sur une perception erronée ou partielle des pratiques ayant cours sur le terrain. Néanmoins, ils nous révèlent la présence du fait religieux au sein du système des soins et les nombreux débats publics qu'il suscite. Selon Bégot (2005), cette visibilité sociale serait une conséquence des reconfigurations du champ religieux, caractérisé par l'effritement des religions instituées, l'émergence de nouvelles formes de spiritualités et de nouvelles manières de croire ainsi que par la diversification sociale et culturelle de la population.

La société québécoise n'échappe pas à ce processus de modification profonde du paysage religieux (Mossière et Deirdre, 2010). En plus de la pertinence de la religion comme symbolique significative pour de nombreux natifs et migrants, ses liens profonds avec la santé laissent entrevoir la place qu'elle occupe dans les rapports de plusieurs personnes aux institutions médicales. À partir d'une recherche menée à Montréal auprès d'intervenants œuvrant dans des Centres de santé et de services sociaux (CSSS) ${ }^{3}$, le présent article porte sur les manifestations du fait religieux dans la relation de soins. Plus spécifiquement, nous tenterons de répondre aux questions suivantes: quelles sont les demandes des patients en matière religieuse et les difficultés attribuables à la diversité religieuse? Quelles sont les réponses des intervenants à ces demandes ou cas difficiles?

Une meilleure compréhension des réactions des professionnels de la santé aux manifestations du religieux est cruciale pour plusieurs raisons. Tout d'abord, considérant les multiples façons dont la religion et la santé s'entrecroisent, la prise en compte ou non des croyances et pratiques religieuses du patient peut avoir une incidence sur la relation de soins. Deuxièmement, l'adaptation des pratiques d'intervention à la diversité religieuse favorise le développement de meilleurs services et de rapports plus inclusifs, venant ainsi renforcer le rôle clé des établissements de santé et de services sociaux dans le processus d'insertion des populations migrantes (Fortin et Le Gall, 2007). Cette capacité à s'ouvrir à la diversité est d'autant plus importante que les personnes nécessitant des soins sont souvent plus vulnérables et dépendantes que celles qui fournissent les soins. Enfin, au Québec, l'adaptation des services de santé à la diversité religieuse fait partie intégrante de la législation. Les établissements ont pour fonction

d'assurer la prestation de services de santé ou de services sociaux de qualité, qui soient continus, accessibles, sécuritaires, et respectueux des droits des personnes et de leurs besoins spirituels et qui visent à réduire ou à résoudre les problèmes de santé et de bien-être et à satisfaire les besoins des groupes de la population. (LSSS, 2006)

On en sait peu toutefois sur la façon dont ils y parviennent et sur l'intérêt porté au fait religieux par les professionnels. Y a-t-il reconnaissance, déni ou contestation de la diversité religieuse dans les interactions quotidiennes entre patients et professionnels? Après avoir brièvement présenté la littérature pertinente sur le sujet et apporté des précisions méthodologiques, nous nous intéressons à la présence du fait religieux dans l'intervention et discutons ensuite de la réponse des professionnels à ces manifestations.

\footnotetext{
${ }^{1}$ La mission des Centres locaux de services communautaires (CLSC) à l'intérieur des CSSS est d'offrir en première ligne à la population de son territoire des services de santé, des services sociaux courants et d'action communautaire, de nature préventive ou curative, de réadaptation ou de réinsertion.

${ }^{2}$ Pour une liste exhaustive et une description plus approfondie des différents cas, voir le rapport final intégral du CCPARDC.

${ }^{3}$ Les CSSS ont pour mandat d'offrir, à proximité du milieu de vie de la population dont ils ont la responsabilité, une large gamme de services de première ligne (services médicaux et sociaux généraux) avec des mécanismes de référence et de suivi pour les services de deuxième et de troisième lignes, soit les services spécialisés et surspécialisés.
} 


\section{Santé, religion et spiritualité}

Plusieurs des travaux en santé ayant intégré la dimension religieuse ont tenté de clarifier les rapports entre religion, spiritualité et santé. Ils suggèrent généralement un lien favorable entre la pratique religieuse et le bienêtre des personnes (voir Chatters 2000; Cochrane, 2006; Lacombe, Pica et Clarkson, 2002; Larson et Larson, 2003). Les attitudes positives, les réseaux de soutien et les comportements prohibés ou encouragés expliqueraient en grande partie une telle incidence (p. ex. Chatters, 2000; Jarvis et Northcott, 1987; Levin et Markides, 1985). Un autre ensemble de textes tente pour sa part de cerner l'influence des évènements de santé, particulièrement des maladies chroniques, sur les croyances et pratiques religieuses ou spirituelles des individus. Ils insistent sur le fait que la plupart des religions offrent un système de signification aidant les individus à affronter les difficultés personnelles (Ferrato et Kelly-Moore, 2002). Par ailleurs, la diversification religieuse de la population dans les sociétés occidentales, laquelle se manifeste par de multiples conceptions religieuses en matière de santé, de maladie et de souffrance, a amené un nombre croissant de chercheurs à étudier les rites de guérison propres à différents groupes religieux (p. ex. Barnes et Sered, 2005; Felix, Levine et Burstin, 2003; Fields, 2001; McGuire, 1988). La religion a également été abordée dans quelques travaux cherchant à saisir la présence et l'importance de la santé et de la médecine dans les organisations religieuses (p. ex. Meintel et Mossière, 2011; Numrich, 2005). De rares études examinent l'impact des croyances religieuses sur la décision des populations natives et immigrantes d'accepter ou non des soins de santé et sur les modes d'utilisation des services (Koenig, 1995; Koenig et Larson, 1998; Koenig, McCullough et Larson, 2001). Elles témoignent par exemple de l'influence de la religion sur la façon dont les immigrants consultent le système des soins et sur leurs prises de décision en matière de santé (Barnes et Sered, 2005; Fadiman, 1998; Ong, 1995). Enfin, d'autres chercheurs ont tenté de voir comment les individus combinent pratiques religieuses et biomédecine dans leurs trajectoires thérapeutiques (Eisenberg et collab., 1993; McGuire, 1988).

\section{Présence de la religion dans les institutions de santé}

Si cette littérature nous renseigne sur les liens multiples entre religion, santé et bien-être, elle nous informe moins sur les rapports pouvant exister entre le religieux et les institutions de soins, particulièrement en contexte de diversité religieuse. Pour Cadge et Fair (2010), qui constatent le manque d'études sur la présence formelle et informelle de la religion dans différents types d'institutions médicales, il importe de saisir comment, quand et sous quelles formes celle-ci apparaît. De même, très peu d'éléments existent sur ce que Bégot (2005) nomme la gestion de la religion, soit la compréhension et la réponse du personnel médical à des aspects rattachés à la religion. De façon générale, les rares travaux qui abordent ces questions traitent du contexte hospitalier et soulignent la faible intégration du fait religieux dans la pratique clinique. Par exemple, dans son excellent travail sur des patients atteints de VIH/sida et leurs médecins en France, Bégot indique que la gestion du fait religieux dans la relation de soins dépendrait de la position occupée par le médecin au sein du champ médical. Les praticiens hospitaliers auraient une approche beaucoup plus distanciée du fait religieux, dans la mesure où l'engagement spirituel du patient remet en question le fondement sur lequel repose la relation de soins (soit le rapport entre expert et profane). Dans ses travaux sur la présence et la construction de la religion et la spiritualité dans de larges centres hospitaliers universitaires américains, Cadge (voir Cadge, 2009; Cadge et Short, 2009) soutient pour sa part que les pédiatres qu'elle a interviewés jugent que, malgré sa pertinence, la religion se situe en dehors de leurs compétences professionnelles et la conçoivent comme une barrière aux soins lorsqu'elle entre en conflit avec le traitement prescrit (Cadge et Short, 2009). Enfin, quelques auteurs, dont certains abordent la diversité religieuse, indiquent que les différences dans les conceptions de la santé, de la maladie et de l'éthique qui peuvent exister entre les personnes croyantes et certains professionnels s'avèrent parfois sources de difficultés dans la rencontre clinique (Buryska 2001; Cochrane, 2006; Pesut et Reimer-Kirkham, 2010).

Nous faisons l'hypothèse que le processus de négociation autour de la diversité religieuse se déroule différemment dans l'intervention de première ligne au Québec, caractérisée par une plus grande proximité quotidienne avec les familles et leurs milieux de vie, mais aussi par une prégnance moindre (en comparaison des services de deuxième et de troisième lignes) des questions de vie et de mort. Même si la variable religieuse occupe, somme toute, une place assez réduite dans le milieu des soins communautaires (Le Gall et Xenocostas, 2011) on y observerait, en dépit des spécificités propres à chacun des secteurs d'intervention, une plus grande reconnaissance des pratiques et croyances religieuses. II existerait donc une multitude de façons de traiter la diversité religieuse dans les 
institutions de soins et ces logiques varieraient selon le mandat de chacune. Pour insister sur le fait que chaque milieu (école, prison, institution de santé, etc.) met en œuvre sa propre logique et que celle-ci déterminerait la forme prise par la diversité religieuse en son sein, Woodhead (2012) utilise l'expression "diversity of diversity».

\section{Méthodologie}

Les données présentées ici ont été recueillies lors d'une recherche exploratoire ${ }^{4}$ portant sur les défis et enjeux posés par la diversité religieuse dans l'intervention de première ligne. Afin de dégager les multiples manifestations que le fait religieux peut revêtir dans les CSSS, 43 entretiens semi-dirigés ont été réalisés auprès de personnes évoluant dans divers programmes et secteurs d'intervention (enfance-famille, soutien à domicile, DI-TED ${ }^{5}$, scolaire, services généraux adultes, maison des naissances, etc.) de plusieurs CSSS montréalais et provenant de différentes disciplines (infirmières, nutritionnistes, psychoéducateurs, psychologues, travailleurs sociaux, sages-femmes, éducateurs, auxiliaires familiaux, organisateurs communautaires, intervenant en soins spirituels) ${ }^{6}$. La plupart des répondants possèdent plusieurs années d'expérience, parfois dans d'autres secteurs d'intervention. Tous les âges sont représentés, bien que la génération des plus de 45 ans prédomine nettement. La majorité des répondants sont des femmes (31/43) et près des deux tiers appartiennent au groupe majoritaire (franco-québécois) (29/43), tandis que les autres sont immigrants (14/43). Les entrevues portent sur les manifestations de la religion dans l'intervention, sur les différentes questions soulevées par ces situations, sur les stratégies et solutions proposées ou adoptées par les intervenants, sur les balises qui guident leurs décisions, sur les outils à leur disposition et sur leur attitude face au fait religieux. Nous cherchions non seulement à prendre connaissance des cas cliniques problématiques, mais aussi à comprendre comment le religieux traverse l'intervention.

\section{Fréquence des manifestations du fait religieux dans la pratique}

Nous n'avons aucunement cherché dans notre étude à quantifier les manifestations du religieux dans la pratique et seuls les cas cliniques ayant retenu l'attention des personnes interviewées ont été recensés. Toutes reconnaissent l'importance de la religion dans le quotidien de plusieurs usagers (ce dont témoignent la tenue vestimentaire ou la présence d'autres signes religieux). Néanmoins, comme Anita, travailleuse sociale dont le travail consiste à favoriser le maintien à domicile des personnes âgées, les intervenants constatent que la plupart d'entre eux, même parmi les plus pratiquants, mettent peu cette dimension de l'avant dans la relation de soins: "Depuis que je travaille, je n'ai pas eu énormément d'aspects religieux qui sont ressortis lors de mes interventions. [...] On travaille avec les personnes âgées et la religion occupe une place importante dans leur vie, mais c'est plus ou moins manifesté lors des entrevues ". Louise, également travailleuse sociale au soutien à domicile, abonde dans le même sens : "Les gens sont très discrets moi je dirais par rapport à la religion. C'est rare qu'on a des gens qui nous en parlent». Pour les personnes qui interviennent lors de moments chargés de sens, comme la fin de vie ou le handicap par exemple, la religion est davantage présente.

Par ailleurs, comme le laisse entendre Mélissa, infirmière en périnatalité qui anime des rencontres prénatales en plus d'offrir un suivi systématique postnatal et du soutien à l'allaitement maternel, les requêtes exprimées au nom de la religion sont plutôt exceptionnelles et rarement formulées comme un ultimatum : " J'ai rarement rencontré quelqu'un qui pensait qu'on allait s'adapter nécessairement. S'ils demandent une adaptation, c'est de façon très gentille, très polie." Les répondants ont d'ailleurs éprouvé des difficultés à documenter un cas clinique problématique associé aux croyances ou pratiques religieuses de leurs patients. La variable religieuse surgit principalement au cours de l'intervention, comme le précise Anita : "Les aspects religieux ça ressort plus tard. II n'y a jamais une demande [...] qui nomme la religion comme un problème. " Quant aux requêtes, elles sont généralement formulées en termes autres que religieux alors que les usagers évoquent rarement leurs croyances ou pratiques religieuses. Le plus souvent, ce sont les professionnels eux-mêmes qui attribuent un rôle à celles-ci.

\footnotetext{
${ }^{4}$ Le projet, intitulé " Diversité religieuse et intervention de première ligne », a bénéficié du financement du CRSH (RDI 2009). L'équipe de chercheurs est composée de Josiane Le Gall, Jacques Rhéaume, Vania Jimenez et Spyridoula Xenocostas. Les entretiens ont été réalisés par Sophie Lemoyne-Dessaint et Amélie Normandin.

${ }^{5}$ Déficience intellectuelle et trouble envahissant du développement.

${ }^{6}$ Nous avons également interviewé 7 intervenants œuvrant dans des organismes communautaires (données non traitées ici).
} 


\section{Exemples de manifestations religieuses}

Les manifestations religieuses concernent une grande variété de contraintes, bien que quelques-unes soient plus récurrentes. Il peut s'agir de croyances, d'interdits, de rituels, de pratiques ou d'obligations. La question des horaires des rendez-vous revient fréquemment et serait même, aux dires de la plupart des intervenants, la principale et souvent l'unique demande. Plusieurs usagers préféreraient consulter en dehors des jours fériés et des célébrations religieuses. Parmi les autres contraintes répertoriées, on note le cas des patients qui souhaitent une nourriture particulière adaptée à leur foi. Par exemple, plusieurs femmes enceintes d'appartenance musulmane consultent la nutritionniste avant de prendre des vitamines prénatales offertes gratuitement par le CSLC, pour s'assurer qu'elles ne contiennent pas de gélatine à base de porc (leur religion leur en interdisant la consommation). Quelques intervenants évoquent aussi la pratique du jeûne : des employés du programme famille/enfance donnent l'exemple des musulmanes qui observent le ramadan pendant leur grossesse, sans y être tenues.

L'intérêt pour la religion se manifeste aussi parfois à travers des tenues vestimentaires particulières, comme dans le cas d'une musulmane assistant à une rencontre de groupe revêtue d'un voile intégral. Une autre situation associée à la religion concerne la demande faite au professionnel de se déchausser avant d'entrer chez un patient lors des visites à domicile. Une intervenante au programme enfance-famille parle de femmes priant lors d'activités de groupes, pratique importunant parfois les autres participants selon elle. Des intervenants scolaires soulignent l'opposition de certains parents aux discussions autour de la sexualité à l'école, notamment en raison de leurs croyances religieuses. Il y a aussi le cas des patients catholiques en perte d'autonomie qui désirent assister à la messe. Des répondants donnent aussi l'exemple des maris répondant systématiquement à la place de leur femme ou celui des demandes de soins prodigués par des femmes professionnelles, demandes attribuées à l'islam. On retrouve encore bien d'autres exemples de manifestations religieuses, dont certains apparaissent dans les pages suivantes.

\section{Réponse des professionnels à la présence du fait religieux dans l'intervention}

Les intervenants semblent bien s'acquitter de l'obligation de respecter la liberté d'expression religieuse de leurs patients. Comme Mélissa, ils affirment tous ajuster facilement leurs pratiques: "C'est certain qu'on n'ira pas faire des changements radicaux, mais il y a des choses qui sont faciles à faire. Il faut juste [...] avoir un peu d'ouverture ». Sandra, une ergothérapeute au soutien à domicile qui a pour tâche d'augmenter la sécurité et l'autonomie fonctionnelle à domicile des personnes ayant des incapacités significatives, procéderait de façon similaire (si un cas survenait) : "Je respecterais ça. J'essaierais justement de l'incorporer autant que possible. Puis de leur donner d'autres options aussi. Si vraiment l'option que je leur propose, c'est contre leur religion ».

Le type d'ajustements mentionnés le plus fréquemment concerne une fois de plus les horaires. Dans le but d'offrir un service adéquat, les répondants tiennent régulièrement compte des fêtes et congés religieux pour fixer l'heure des rendez-vous. Pour Marie, psychologue employée aux services généraux adultes, éviter de se rendre chez un patient un jour férié relève de la "finesse sociale ». De son côté, Suzie, intervenante au programme enfancefamille, s'informe toujours du meilleur moment pour rencontrer à leur domicile les mères qui viennent d'accoucher, ce qu'elles apprécient : «On essaie de toujours vérifier auprès des gens pour dire : "est-ce que c'est le bon temps pour aller chez vous?" ou "vous pouvez venir ici?", alors de ce côté-là, je pense que les clients apprécient notre respect de leur coutume et de leur religion. " Pour satisfaire la demande de plusieurs musulmanes, les infirmières du programme enfance-famille vérifient la composition des vitamines prénatales ou, comme Mélissa, en commandent sans gélatine: "Ça a été fait, parce que c'était tellement simple à faire ». Si quelques intervenants considèrent risqué pour leur sécurité de se déchausser au domicile des patients, d'autres, comme Miranda, une travailleuse sociale travaillant auprès de personnes âgées en perte d'autonomie et en perte cognitive, n’hésitent pas à le faire ou alors transportent avec eux une seconde paire de souliers.

Pour les personnes interviewées, offrir des soins adaptés relève d'une de leurs responsabilités, qui consiste à respecter le caractère unique de l'individu et à le considérer dans sa globalité. Dans le contexte de la prestation de soins et de services, faire la promotion des meilleurs intérêts d'un individu peut signifier avoir à respecter une diversité d'opinions, de croyances, de pratiques et de préférences personnelles. Les professionnels tentent donc de proposer des solutions respectueuses de la liberté de religion de l'usager, souvent en position de dépendance et de 
vulnérabilité. Ils estiment aussi que des aménagements mineurs peuvent renforcer la relation de soins. À leurs yeux, les demandes liées à la religion et les obstacles qu'elle peut générer ne représentent qu'une dimension parmi d'autres de la vie du patient dont ils doivent tenir compte.

L'expression « être attentif au sens donné par le client » revient fréquemment dans les entretiens. Selon Anita, il est impératif de laisser la personne devant soi s'exprimer pour mieux saisir la complexité de la situation et de s'adapter: "Au début, la première intervention c'est de les écouter, d'avoir le plus d'éléments possible pour pouvoir bien analyser la demande. " De même, pour Angela, une éducatrice au programme enfance-famille, la religion constitue un élément parmi d'autres à intégrer dans les soins : " C'est pertinent parce que c'est quelque chose qui fait du sens pour eux. À ce moment-là, je pense qu'on ajuste, mais ce n'est pas parce que c'est la religion. C'est parce que ça a une importance au niveau de l'intervention ». Elle poursuit en indiquant qu'adapter l'intervention aux pratiques religieuses va de soi: "Je pense que ça se fait naturellement [...] Parce que c'est comme si on est tout le temps en train de s'adapter et d'échanger quand on travaille en individuel. Alors, on dirait que le religieux, ça passe avec ça. »

\section{Limites à l'adaptation des pratiques}

La question qui se pose aux intervenants ne consiste donc pas à décider s'ils doivent adapter leurs pratiques ou pas, mais plutôt jusqu'où et comment ils doivent le faire. Pour y répondre, plusieurs éléments entrent en ligne de compte, notamment leurs mandats professionnels et celui de leurs équipes, les orientations de l'établissement, les politiques de santé publique ou encore les grands principes de droit. La loi limite l'adaptation des services aux possibilités des ressources humaines, matérielles et financières des établissements de santé de répondre à la demande. On note ainsi le souci des répondants de ne pas adapter les soins au détriment des possibilités de l'offre des soins de santé globale. Sandra, qui trouve facile en général de répondre aux rares demandes rencontrées, rejetterait celles qui entraîneraient des coûts indus ou qu'elle jugerait non équitables : " Il faut penser aussi à l'universalité des soins. S'il y a une personne qui veut certaines choses pour sa religion, mais ça coûte 4 fois plus cher, c'est sûr qu'il faut prendre ça en considération, si on veut qu'il y ait des services pour tout le monde ».

La sécurité des employés et celle des patients, de même que l'application des règles d'hygiène et d'asepsie, servent également de balise aux adaptations. Invitée à énumérer les facteurs qu'elle prendrait en compte pour ajuster ou pas ses pratiques, Anita, parle notamment des conséquences de refuser un ajustement sur la santé du patient: "Est-ce que la personne est en danger si on ne répond pas à sa demande? C'est l'élément principal sur lequel on se base. [...] C'est quoi l'impact du rejet de sa demande? " La sécurité du patient demeure aussi en tout temps une des priorités de Jean, un travailleur social en milieu scolaire qui travaille en complémentarité et en soutien aux enseignants afin de répondre aux besoins des jeunes: "Est-ce que ce que je vois ça, je peux objectivement dire que c'est négatif pour la personne? De façon générale, l'enjeu est souvent là. » Pour Louis, un intervenant en soins spirituels dans un centre d'hébergement et exerçant des activités de soutien et d'accompagnement à la vie spirituelle et religieuse des usagers, à leur famille ainsi qu'à leurs proches, ajuster sa pratique ne pose pas problème "pourvu que la sécurité du résident, des autres et des employés soit assurée. " II donne l'exemple suivant pour illustrer son propos: "Par exemple, on n'allumerait pas de chandelles dans la chambre d'un mourant pour des motifs religieux. " Dans son travail d'infirmier en services généraux, qui consiste en gros à donner des conseils et les soins requis à la suite d'une blessure, d'une maladie chronique ou d'une chirurgie, Robert est amené régulièrement à se rendre au domicile des patients pour changer des pansements. S'il tient compte le plus souvent des congés religieux pour fixer un rendez-vous, cet infirmier refuse de faire des concessions lorsqu'un pansement postchirurgical doit être refait quotidiennement et que les risques d'infection sont élevés. Pour les mêmes raisons, il refuserait de modifier sa façon de procéder: "II n'y a aucun compromis non plus, pour quelqu'un qui voudrait qu'on [fasse] un pansement d'une telle manière, avec telle autre chose, si ça peut compromettre entre autres l'asepsie, la stérilité, des choses comme ça, la porte d'entrée des microbes. »

La mission et la philosophie des établissements influencent également la prise de décision des intervenants, comme en témoigne Angela à propos des éléments qu'elle intègre pour évaluer une demande pour des motifs religieux : " Je pense aux objectifs de l'activité, aux valeurs du CLSC. Est-ce que la base de mon travail? Est-ce que ça vient entraver ou pas? Si ça n'entrave pas, il n'y a pas de problème. Je pense qu'on peut accommoder tout le 
monde. " Pour elle, toute adaptation qui remettrait en question l'intervention serait rejetée. Dans le cadre de son travail, il lui arrive d'animer des activités de groupe qui, selon elles, se prêtent davantage à la controverse que les rencontres individuelles. C'est lors d'une telle activité qu'a surgi le seul cas problématique de sa carrière: une musulmane s'est dite choquée de la présence d'un père et a demandé à ce qu'il soit exclu du groupe, ce qui a été catégoriquement refusé, car en contradiction avec l'objectif de l'activité, qui est de «travailler les compétences parentales, autant chez les mères que les pères ».

Quel soutien les intervenants reçoivent-ils de leur institution dans la gestion de la diversité religieuse? Existent-ils des ressources pour les aider à prendre une décision éclairée? Dans les CSSS montréalais à l'étude, le fait religieux est très peu intégré à la culture organisationnelle. II n'existe aucun guide ou politique sur la diversité religieuse. Dans un établissement, cette dimension est intégrée dans une politique sur la diversité culturelle. Cela dit, lorsqu'ils rencontrent un problème touchant au fait religieux, les intervenants peuvent s'appuyer sur différentes instances telles que la direction de la qualité ou encore le comité d'éthique clinique. Dans les faits, les personnes interviewées qui consultent disent se tourner principalement vers des collègues qui ont davantage d'expérience dans ce domaine ou encore vers leurs supérieurs (directeur, chef de service, chef de programme, coordonnateur clinique, conseiller clinique, etc.). Les propos de Chantal (travailleuse sociale) sur les ressources disponibles au niveau de son établissement résument bien les démarches entreprises par plusieurs intervenants lorsque survient un cas problématique :

Ça, il n'y en a pas beaucoup [des ressources]. C'est sûr quand on a des questionnements, des fois, on ne sait plus comment agir dans une situation $X$. On peut aller voir les supérieurs, on peut aller voir en supervision s'il peut y avoir quelqu'un... Si dans nos collègues, on pense que les gens qui sont plus masters ou qui ont le plus d'expérience, qui peuvent nous venir en aide dans les dossiers complexes ou question pointue, religieuse, je pense qu'il y a une place pour en parler.

Pour obtenir du soutien, plusieurs intervenants nous ont également dit compter principalement sur les réunions d'équipe, en particulier lors des discussions de cas, comme les extraits suivants en témoignent :

Le soutien en fait, c'est surtout... Ce sont les réunions vraiment notre seul soutien, parce qu'on met nos connaissances en commun, et on essaie de trouver une solution avec les connaissances de chacun, et voir comment on peut nous adapter. (Miranda, travailleuse sociale)

On parle entre nous. Nous sommes une équipe avec beaucoup, beaucoup d'expérience. Tu sais entre nous, de 30 à 35 ans d'expérience chacun. Et alors on se parle. (Emma, infirmière)

\section{Dialogue et solutions de compromis}

Les professionnels affirment qu'il y a toujours place, à travers le dialogue et la négociation, à des solutions de compromis qui respectent les principales règles de soins de santé et qui tiennent compte des attentes des usagers. D'ailleurs, rares sont les exemples de refus de collaborer de la part du patient. Selon Sandra, l'ouverture mutuelle (intervenant/usager) explique l’absence de difficultés: "Ça n’a jamais été majeur, il y a toujours une flexibilité qui est possible autant d'un côté que de l'autre ». Louis, cité plus haut, proposerait des "petits lampions électriques » à la place des bougies: "C'est de cette façon-là qu'on procéderait parce que, avec les chandelles, la sécurité serait mise en danger, mais d'un autre côté le besoin de lumière, de veilleuse, on peut le faire d'une autre façon. " Une infirmière en périnatalité souligne pour sa part qu'elle informe toujours les parents des risques associés à la pratique qui consiste à placer certains objets (épinglette, collier, croix, livre, etc.) dans le lit du nourrisson en guise de protection :

Il faut que je leur dise que ce n'est pas tout à fait sécuritaire, qu'il faut faire attention. Peut-être le mettre juste quand vous êtes là, mais peut-être l'enlever quand l'enfant dort. [...] Puis je leur dis toujours: "Je le sais que ça fait partie de votre culture, de votre religion, je veux juste vous aviser".

De son côté, Robert rencontre régulièrement des patientes musulmanes dont les maris exigent un suivi par une infirmière lors d'un changement de pansement. Il tente de répondre à leur demande dans la mesure du possible, « mais s'il n’y en a pas [de solutions], il n’y en a pas » et il doit alors négocier: "Parfois, ça arrive que je sois le seul infirmier disponible, alors il faut vraiment négocier et essayer d'organiser quelque chose. Mais avec le temps, on développe des stratégies. " Pour rassurer et gagner la confiance des maris, il leur demande d'assister à I'intervention et leur explique comment procéder ou encore il les invite à participer : 
Je lui dis "donne-moi telle sorte de pansement, donne-moi ci, donne-moi ça" [...] Alors il voit que c'est scientifique de A à Z, et que ce n'est que ça. Et par la suite, on gagne une confiance, à force de faire ces mêmes traitements-là, parce que des pansements, des fois, il faut les faire tous les jours, et tu en as pour trois semaines, un mois. Alors à un moment donné, au bout de 3-4 fois, ça va très bien.

\section{Prise en compte du fait religieux dans l'intervention}

Compte tenu des multiples éléments qui entrent en ligne de compte dans l'analyse d'une situation, déterminer la place du religieux s'avère souvent une tâche ardue. Il est possible de penser qu'une situation imputée à la religion relève d'autres facteurs, comme la méconnaissance des services par les usagers, leur parcours migratoire ou encore leurs statuts. Lorsqu'on demande à Sandra si elle a déjà rencontré des requêtes de la part de ses patients fondées sur des motifs religieux, elle déclare : "C'est que je ne le sais pas, jusqu'à quel point c'est religieux non plus ». De plus, de l'aveu de plusieurs, une confusion persiste très souvent entre religion et culture. Selon quelques personnes interviewées, tout rejeter sur la religion et évacuer les autres explications est un réflexe facile et plutôt fréquent. Miranda donne l'exemple d'un dossier qui lui a été transféré en raison de son expertise sur l'islam, mais où la religion ne jouait aucun rôle :

Quand je suis allée voir la madame, c'est vrai, en fait, elle est musulmane, mais elle avait des problèmes de troubles de personnalité non diagnostiqués [...] Et pour moi c'était étonnant de voir qu'on n'avait pas considéré les problèmes de santé mentale, on avait vu juste le problème de religion. La religion n'y était pour rien.

La question qui se pose est donc de savoir si les intervenants explorent la dimension religieuse avec les usagers, plus particulièrement lorsqu'ils considèrent qu'elle constitue une des dimensions de la relation de soins. L'appartenance religieuse ne fait pas partie des informations recueillies par les établissements. Seule une section du formulaire d'évaluation de l'autonomie multiclientèle utilisé dans certains secteurs d'intervention permet aux intervenants de se renseigner sur les croyances et valeurs spirituelles des patients. Cela dit, plusieurs d'entre eux nous disent omettre de remplir cette section. En fait, comme nous l'illustrons ailleurs (Le Gall et Xenocostas, 2011), le fait religieux est peu intégré dans une telle relation puisque très peu d'intervenants en traitent spontanément ou l'explorent en détail avec leurs patients. Seules quelques personnes, principalement des immigrants ou des intervenants pratiquants, se disent à l'aise avec les croyances et pratiques religieuses et questionnent leurs patients sur le sujet, mais uniquement après l'établissement d'une relation de confiance. La religion se situe donc en dehors des compétences des intervenants et ne devient une dimension de l'intervention que si elle occupe une place importante dans la vie des usagers. Les intervenants sont plus ou moins au fait de différentes pratiques religieuses. Selon Marie, ils devraient pouvoir explorer la dimension religieuse avec les usagers : « Un défi, ce serait peut-être accompagner les intervenants à être plus à l'aise, à poser juste une question : comment tu expliques I'handicap, les difficultés que tu vis selon ta religion? " Elle garde néanmoins une certaine réserve: "Mais il faut savoir si la personne devant toi est croyante ou non. " Pour Mélissa, un des principaux défis que pose la diversité religieuse au personnel est de bien comprendre les enjeux: «Des fois, on porte des jugements basés sur des renseignements faux, ou des choses qu'on croit, mais qui ne sont pas véridiques. [...] Des gestes que nos clients posent ou des choses qui suivent, mais dont on ne comprend pas la raison. »

Par ailleurs, quelques répondants avouent ressentir une incompréhension devant différentes pratiques. D’autres disent éprouver parfois des difficultés à concilier les valeurs d'intervention ou leurs valeurs personnelles avec les valeurs et pratiques de l'usager. Les questions de l'autonomie et celle de l'égalité homme/femme reviennent régulièrement dans les témoignages, en particulier dans ceux des intervenantes. Une musulmane enceinte qui demande à être suivie par une femme obstétricienne ou encore un mari qui répond à la place de sa femme sont des exemples classiques de comportements qui les choquent. Mélissa explique que les dimensions religieuses viennent parfois interpeller ses valeurs personnelles, principalement au niveau des relations homme/femme :

Ça vient de mon féminisme à moi, puis moi, ici je peux faire tout ce que je veux. Je peux conduire une voiture puis je peux sortir quand je veux, puis je peux montrer mon visage, je peux aller me baigner! Je peux aller... Ça va loin quand on parle de religion. D'avoir une cliente, il fait $35^{\circ} \mathrm{C}$ dehors puis elle ne peut pas enlever de pièces de vêtement. Ça, je trouve ça... il y a des fois que je trouve ça dur. Mais comme je te dis, ça va rarement affecter à un point tel la santé de l'enfant ou la mère pour que je puisse dire : "Bien non, ça n'a pas de bon sens". 
De plus, les personnes interviewées soulignent que leur relative ouverture face au religieux est loin d'être partagée par les autres membres de leur équipe. Marie dit que le malaise de ses collègues est palpable, d'où une vive réaction de leur part lorsqu'elle a accepté de participer à la recherche: «Quand je suis dans des réunions avec $\mathrm{d}^{\prime}$ autres personnes [...] on ne mentionne pas du tout la religion [...] on ne parle pas facilement de religion $»$.

\section{Conclusion}

Les divers cas cliniques problématiques associés aux croyances ou pratiques religieuses médiatisées lors de la crise des accommodements raisonnables laissent croire au caractère récurent des manifestations du fait religieux dans le milieu de la santé au Québec. Dans notre étude auprès d'intervenants de la première ligne, nous avons observé une réalité tout autre. Aux dires des personnes interviewées, les patients évoquent très peu d'éléments religieux dans la relation de soins, tout comme ils ne formulent qu'exceptionnellement des exigences liées à des prescrits religieux. De plus, la grande majorité des situations où apparaît la variable religieuse se règle bien, à la convenance de tous. Pour les intervenants, qui privilégient une approche globale dans leur travail, la religion constitue une dimension parmi d'autres de la vie du patient, ce qui justifie des adaptations au quotidien de leur part. Un tel constat vient corroborer les propos de certains auteurs qui insistent sur l'aptitude des intervenants à appréhender la singularité de chaque situation et à ajuster leur intervention en conséquence (Cloutier, 2011; Racine, 2007).

Une telle logique de reconnaissance des différences religieuses dans les interactions quotidiennes entre patients et professionnels relève de la mission des institutions de santé, qui est de prendre soin des patients. La nature des interventions en première ligne diffère toutefois de celles rencontrées en milieu hospitalier, d'où une évocation moins fréquente d'éléments religieux dans la relation de soins. La tâche des professionnels qui y œuvre est moins d'administrer des médicaments ou de proposer des traitements médicaux. Les moments chargés de sens pour les patients et leurs familles, plus propices à la manifestation de la religion, comme lors de problèmes de santé sérieux qui nécessitent souvent des prises de décisions médicales, y sont moindres. La nature des activités des hôpitaux, orientées davantage vers les soins curatifs que vers des actions de prévention, peut expliquer la préoccupation des médecins face aux pratiques susceptibles d'interférer avec leurs prescriptions thérapeutiques ou leurs traitements (Bégot, 2005; Cadge et Short, 2009). Cela dit, chaque secteur d'intervention au CSSS présente ses propres spécificités et certains, de par la nature des services offerts, sont plus concernés par le fait religieux que d'autres, comme les programmes des soins palliatifs ou DI-TED, où il serait pertinent de réaliser des études plus approfondies sur la question.

Alors que la gestion du pluralisme religieux a été principalement abordée à partir de la perspective des États nations et des gouvernements, l'examen de la place de la religion dans les institutions de santé, plus spécifiquement dans les pratiques et interactions quotidiennes, apporte un nouvel éclairage aux processus de négociation autour de la diversité religieuse, tout en permettant de saisir comment les individus vivent leur religion au quotidien en dehors des lieux de culte (Cadge et Ecklund, 2007; Orsi, 2003). Le défi consiste toutefois à déterminer comment et par qui sont dictées les différentes logiques à l'œuvre dans le traitement de la différence religieuse. Dans le cas qui nous intéresse, il semble que le processus de prise en compte des différences religieuses, encore à ses balbutiements, soit défini en grande partie par les structures de santé elles-mêmes. Nous avons montré, rapidement, qu'en plus d'être exclue de la culture organisationnelle des institutions, la question religieuse est très peu abordée par les intervenants et qu'elle suscite un certain malaise chez plusieurs. Rares sont ceux qui interrogent leurs patients sur leurs croyances et pratiques religieuses ou encore qui abordent le fait religieux de façon systématique dans leur travail. Un tel malaise est à associer au contexte plus large, alors que la religion reste un sujet délicat au Québec qui cristallise les tensions. II n’est qu'à rappeler encore une fois les débats publics qui ont récemment eu lieu autour des accommodements religieux et qui ont mis au jour les inquiétudes au sein de la population québécoise quant à ses questionnements identitaires. À cet égard, il est intéressant de noter que la majorité des cas cliniques associés à la religion documentés lors de notre recherche focalisent l'attention sur les populations musulmanes. Les controverses et conflits publics sur l'islam sont très présents au Québec, où la perception des musulmans se révèle particulièrement négative au sein d'une fraction de la population (Helly, 2005). Il est à noter que les intervenantes manifestent une plus grande sensibilité pour la question de la religion et de l'égalité homme-femme que les intervenants. De même, les personnes ayant elles-mêmes vécu l'expérience du processus migratoire semblent plus à l'aise avec le fait religieux. D’autre part, si nos donnés montrent que, dans 
l'ensemble, les intervenants des CSSS s'ajustent facilement aux rares requêtes exprimées au nom de la religion, il existe des limites aux adaptations, parmi lesquelles figurent les normes d'intervention (par ex. autonomie, primauté de l'individuel sur le collectif, indépendance), qui sont loin d'être neutres, comme le soulignent plusieurs auteurs (Fortin, 2008; Fox, 2005; Taylor, 2003). Il y a déjà quelques années, Massé (1999) qualifiait la santé publique d'entreprise de normalisation des comportements reliés à la santé procédant « en amenant le citoyen postmoderne individualisé et rationalisé à intérioriser les normes » (1999, p. 12)

Pour terminer, soulignons qu'il n'existe pas de réponses toutes faites ou de recettes s'appliquant à certaines situations précises impliquant la religion. Pour comprendre l'ensemble des besoins exprimés par les patients et adapter les services du mieux possible, les intervenants doivent reconnaître la spécificité de chacun, incluant leurs propres valeurs et croyances personnelles. Ils ont l'obligation de répondre aux demandes du patient tout en maintenant un équilibre entre leurs normes professionnelles de pratiques, celles de leur équipe, les politiques publiques et les attentes du patient, ce qui constitue en soi un réel défi. Une analyse professionnelle complète des situations difficiles doit donc reposer sur un questionnement sur les aspects cliniques, culturels/religieux et éthiques. Surtout, il est essentiel de garder en tête que l'attribution à tort d'une situation à la variable religieuse comporte le danger, en s'attardant, comme dans le cas de la culture (Castaneda, 2010), aux normes d'un groupe, de passer sous silence le "rôle pathogénique " des inégalités sociales (Hirsch, 2003), de la pauvreté et de l'exclusion sociale (Farmer, 2003). La religion n'étant souvent qu'un enjeu parmi d'autres dans la rencontre clinique, pas nécessairement centrale dans la situation médicale du patient, il s'avère donc important d'insister sur l'ensemble des dimensions qui s'y juxtaposent (p. ex. pays d'origine, parcours migratoire, culture, classe sociale, genre, niveau d'éducation, degré de familiarité avec les normes bureaucratiques/organisationnelles/ déontologiques du système de santé, rôles et mandats du professionnel). D'autre part, l'absence de prise en compte d'éléments religieux dans l'intervention peut amener les professionnels à interpréter les cas cliniques en fonction de leurs propres valeurs. L'enjeu devient, alors, d'arriver à cerner la juste place à accorder au fait religieux dans la relation de soins, ce qui exige une bonne communication entre professionnels et usagers et, ultimement, une meilleure compréhension des cas cliniques.

\section{Références bibliographiques}

Barnes, L. et Sered, S. (dir.) (2005). Religion and healing in America. New York : Oxford University Press.

Bégot, A.-C. (2005). La gestion du fait religieux au sein de l'institution médicale. Perspective historique et étude de cas : des patients touchés par le VIH/Sida et leurs médecins (rapport de recherche). Association Sidaction.

Buryska, J. (2001). Assessing the ethical weight of cultural, religious and spiritual claims in the clinical context. Journal of Medical Ethics, 27, 118-122.

Cadge, W. (2009). Prayers in the clinic: How pediatricians and pediatric oncologists respond. Southern Medical Journal, 102(12), 1218-1221.

Cadge, W. et Ecklund, E. (2007). Immigration and religion. Annual Review of Sociology, 33(1), 359-379.

Cadge, W. et Fair, B. (2010). Religion, spirituality, health and medicine : sociological intersections. Dans C. Byrd, Allan Fremont, S. Timmermans et P. Conrad (dir.), Handbook of Medical Sociology (p. 341-362). Vanderbilt University Press.

Cadge, W. et Short, N. (2009). Religion and spirituality : A barrier and a bridge in the everyday professional work of pediatric physicians. Social Problems, 56(4), 702-721.

Castaneda, H. (2010). Im/migration and health: conceptual, methodological, and theoretical propositions for applied anthropology. Napa Bulletin, 34, 6-37.

Chatters, L. (2000). Religion and health: Public health research and practice. Annual Review of Public Health, 21, 335-367.

Cloutier, G. (2011). La valorisation des savoirs de femmes immigrantes en milieu communautaire. Source d'inspiration pour l'intervention sociale. Montréal : Éditions Vézina.

Alterstice - Revue Internationale de la Recherche Interculturelle, vol. $2, n^{\circ} 2$ 
Cochrane, J. (2006). Religion in the health of migrant communities: Asset or deficit ? Journal of Ethnic and Migration Studies, 32(4), 715-736.

Eisenberg, D., Kessler, R., Foster, C., Norlock, F., Calkins, D. et Delbanco, T. (1993). Unconventional medicine in the United States - Prevalence, costs, and patterns of use. New England Journal of Medicine, 328, 246-252.

Fadiman, A. (1998). The spirit catches you and you fall down: A Hmong child, her American doctors and the collision of two cultures. New York : Noonday.

Farmer, P. (2003). Pathologies of power: Health, human rights, and the new war on the poor. Berkeley : University of California Press.

Felix A., Levine, D. et Burstin, H. (2003). African American church participation and health care practices. Journal of General Internal Medicine, 18, 908-913.

Ferrato, K. et Kelley-Moore, J. (2002). Religious consolation among men and women : do health problems spur seeking. Journal for the Scientific Study of Religion, 39(2), 220-234.

Fields, G. (2001). Religious therapeutics : Body and health in yoga, ayurveda, and tantra. Albany : SUNY Press.

Fortin, S. (2008). The paedeatric clinic as negociated social space. Anthropology and Medicine, 15(3), $175-187$.

Fortin, S. et Le Gall, J. (2007). Néonatalité et constitution des savoirs en contexte migratoire : familles et services de santé. Enjeux théoriques, perspectives anthropologiques. Enfances, Familles, Générations, 6, 16-37.

Fox, R. (2005). Becoming a physician: Cultural competence and the culture of medicine. New England Journal of Medicine, 353(13), 316-319.

Helly, D. (2005). La gestion de la différence religieuse au Canada et le cas de l'islam. Revue marocaine d'études internationales, 13, 57-83.

Hirsch, J. (2003). Anthropologists, migrants, and health research: Confronting cultural appropriateness. Dans N. Foner (dir.), American Arrivals : Anthropology Engages the New Immigration (p. 229-257). Santa Fe : School of American Research Press.

Jarvis, G. et Northcott, H. (1987). Religion and differences in morbidity and mortality. Social Science and Medecine, $25,813-824$.

Koenig, H. (1995). Use of acute hospital services and mortality among religious and non-religious copers with medical illness. Journal of Religious Gerontology, 9(3), 1-21.

Koenig, H. et Larson, D. (1998). Use of hospital services, religious attendance, and religious affiliation. Southern Medical Journal, 91(10), 925-932.

Koenig, H., McCullough, M. et Larson, D. (2001). The handbook of religion and health. New York : Oxford University Press.

Lacombe, H., Pica, L. et Clarkson, M. (2002). Spiritualité, religion et santé chez des immigrants récents : une approche exploratoire. Dans Institut de la Statistique, Santé et bien-être, immigrants récents au Québec: une adaptation réciproque? Étude auprès des communautés culturelles, 1998-1999 (chap. 17, p. 319-332). Québec : Institut de la statistique du Québec.

Larson, D. et Larson, S. (2003). Spirituality's potential relevance to physical and emotional health : A brief review of quantitative research. Journal of Psychology and Theology, 31(1), 37-51.

Le Gall, J. et Xenocostas, S. (2011). L'adaptation des soins sociosanitaires de première ligne à la diversité religieuse : l'exemple du Québec. Ethnologies, 33(1), 169-189.

Levin, J. et Markides, K. (1985). Religion and health in Mexican Americans. Journal of Religion and Health, 24(1), 60-69.

Loi sur les services de santé et les services sociaux (LSSS) (2006). L.R.Q. c. S-4.2, art $100: 87$. 
Massé, R. (1999). La santé publique comme nouvelle moralité. Cahiers de Recherches éthiques, 22, $155-176$.

McGuire, M. (1988). Ritual healing in suburban America. New Brunswick, NJ : Rutgers University Press.

Meintel, D. et Mossière, D. (2011). Tendances actuelles des rituels, pratiques et discours de guérison au sein des groupes religieux contemporains. Quelques réflexions. Ethnologies, 33(1), 5-18.

Mossière, G. et Meintel, D. (2010). Tradition and transition : Immigrant religious communities in urban contexts (Québec). Dans R. D. Hecht et V. F. Biondo (dir.), Religion in the practice of daily life (p. 481-508). Santa Barbara, CA : Praeger.

Numrich, P. (2005). Complementary and alternative medicine in America's "two Buddhisms". Dans L. Barnes et S. Sered (dir.), Religion and healing in America (p. 343-357). New York : Oxford University Press.

Pesut, B. et Reimer-Kirkham, S. (2010). Situated clinical encounters in the negotiation of religious and spiritual plurality: A critical ethnography. International Journal of Nursing Studies, 47(7), 815-825.

Ong, A. (1995). Making the biopolitical subject : Cambodian immigrants, refugee medicine and cultural citizenship in California. Social Science and Medecine, 40, 1243-1257.

Orsi, R. (2003). Is the study of lived religion irrelevant to the world we live in? Special presidential plenary address. Journal for the Scientific Study of Religion, 42(2), 169-174.

Racine, G. (2007). De la production du silence aux invitations à l'échange de savoirs. Le cas des pratiques en travail social. Dans H. Dorvil (dir.), Problèmes sociaux - Tome IV. Montréal : Presses de l'Université du Québec.

Taylor, J. (2003). The story catches you and you fall down: Tragedy, ethnography, and "cultural competence". Medical Anthropology Quarterly, 17(2), 159-181.

Woodhead, L. (2012, 15-16 fév). Spirituality and the institutionalisation of religious pluralism. Communication présentée au colloque From religious diversity to religious pluralisms. What is at stake?, Padoue, Italie. 